\title{
The Irregularity of Some Composite Graphs
}

\author{
Nilanjan De ${ }^{1} \cdot$ Anita Pal $^{2} \cdot$ Sk. Md. Abu Nayeem ${ }^{3}$
}

\begin{abstract}
The irregularity of a simple undirected graph $G=(V, E)$ is defined as $\operatorname{irr}(G)=$ $\sum_{u v \in E(G)}\left|d_{G}(u)-d_{G}(v)\right|$, where $d_{G}(u)$ is the degree of the vertex $u$. This graph invariant is also known as third Zagreb index. In this paper, we investigate how the irregularity of a graph changes with various subdivision operations. Moreover, we find some exact expressions for irregularity of different composite graphs such as double graph, double cover graph, generalized thorn graph and subdivision vertex corona of graphs.
\end{abstract}

Keywords Vertex degree · Graph invariant · Zagreb indices · Graph irregularity · Composite graph $\cdot$ Graph operations

Mathematics Subject Classification Primary 05C35 · Secondary 05C07, 05C40

\section{Introduction}

Let $G=(V, E)$ be a simple undirected graph with $n$ vertices and $m$ edges. We denote the degree of a vertex $u$ by $d_{G}(u)$ and the maximum and minimum degree of the graph $G$ by $\Delta$ and $\delta$ respectively. The imbalance of an edge $e=u v \in E(G)$ is defined as $\operatorname{imb}(e)=$ $\left|d_{G}(u)-d_{G}(v)\right|$. In [2], Albertson defined the irregularity of $G$ as

Nilanjan De

de.nilanjan@rediffmail.com

Anita Pal

anita.buie@gmail.com

Sk. Md. Abu Nayeem

nayeem.math@aliah.ac.in

1 Department of Basic Sciences and Humanities (Mathematics), Calcutta Institute of Engineering and Management, Kolkata 700 040, India

2 Department of Mathematics, National Institute of Technology, Durgapur 713 209, India

3 Department of Mathematics, Aliah University, New Town, Kolkata 700 156, India 


$$
\operatorname{irr}(G)=\sum_{e \in E(G)} i m b(e)=\sum_{u v \in E(G)}\left|d_{G}(u)-d_{G}(v)\right|
$$

and found upper bounds of irregularity for bipartite graphs, triangle-free graphs and a sharp upper bound of irregularity for trees. Also, Hansen and Mélot [15] characterized the graphs with $n$ vertices and $m$ edges with maximal irregularity. A lot of researches on irregularity of graphs have been carried out in the recent past [1,4,20,21].

It is clear that the irregularity of a graph is always nonnegative and for a regular graph $G, \operatorname{irr}(G)=0$. We recall that the first and the second Zagreb indices of a graph $G$, denoted by $M_{1}(G)$ and $M_{2}(G)$ respectively, are one of the oldest topological indices introduced by Gutman and Trinajstić [13] and were defined as

$$
\begin{aligned}
& M_{1}(G)=\sum_{u \in V(G)} d_{G}(u)^{2}=\sum_{u v \in E(G)}\left[d_{G}(u)+d_{G}(v)\right] \text { and } \\
& M_{2}(G)=\sum_{u v \in E(G)} d_{G}(u) d_{G}(v) .
\end{aligned}
$$

In [12], Fath-Tabar named the sum (1) as the third Zagreb index and denoted it by $M_{3}(G)$ and presented some upper and lower bounds of $M_{3}(G)$ in terms of $M_{1}(G)$ and $M_{2}(G)$. However, we shall refer this sum as irregularity of graphs throughout this paper.

In this paper, we will show how the irregularity of a graph changes with the various subdivision operations. Moreover, we give exact expressions for irregularity of double graph, extended double cover graph, generalized thorn graphs and subdivision vertex corona of graphs.

\section{Irregularity of Some Subdivision Graphs}

Let $G$ be a connected graph. The line graph of $G$, denoted by $L(G)$ is the graph whose vertices are the edges of $G$ and two vertices of $L(G)$ are adjacent if and only if the corresponding edges are adjacent in $G$.

In the following, we study the irregularities of some subdivision graphs which are defined below and were investigated for their Wiener and hyper-Wiener indices in [9, 11,24].

Definition 1 The subdivision graph of a graph $G$ denoted by $S(G)$ is obtained from $G$ by replacing each edge of $G$ by a path of length two.

Definition 2 The triangle parallel graph of a graph $G$ is denoted by $R(G)$ and is obtained from $G$ by replacing each edge of $G$ by a triangle.

Definition 3 The line superposition graph $Q(G)$ of a graph $G$ is obtained from $G$ by inserting a new vertex into each edge of $G$, and joining with edges each pair of new vertices on adjacent edges of $G$.

Definition 4 The total graph $T(G)$ of a graph $G$ has its vertices as the edges and vertices of $G$. Adjacency in $T(G)$ is determined by adjacency or incidence of the corresponding elements of $G$.

In [22], Tavakoli et al. have shown that $\operatorname{irr}(L(S(G)))=\operatorname{irr}(G)$. Here, we first determine the lower bound of irregularity of the subdivision graph $S(G)$ of $G$ in terms of the number of edges and the first Zagreb index of $G$. 
Theorem 1 The irregularity of the subdivision graph $S(G)$ of $G$ follows the inequality $\operatorname{irr}(S(G)) \geq M_{1}(G)-4 m$, and equality holds if and only if all the vertices of $G$ are of degree more than one.

Proof The subdivision graph $S(G)$ is obtained from $G$ by replacing each edge by a path of length two. So, $|V(S(G))|=n+m$ and $|E(S(G))|=2 m$. Also, $d_{S(G)}(v)=d_{G}(v)$, if $v \in V(G)$, otherwise $d_{S(G)}(v)=2$. Hence the irregularity of $S(G)$ is given by

$$
\begin{aligned}
\operatorname{irr}(S(G)) & =\sum_{u v \in E(S(G))}\left|d_{S(G)}(u)-d_{S(G)}(v)\right| \\
& =\sum_{u \in V(S(G))}\left|d_{S(G)}(u)-2\right| \\
& =\sum_{u \in V(G)}\left|d_{G}(u)-2\right| d_{G}(u) .
\end{aligned}
$$

Now since for any two positive real numbers $a$ and $b,|a-b| \geq|a|-|b|$, from above we have

$$
\operatorname{irr}(S(G)) \geq \sum_{u \in V(G)}\left(d_{G}(u)-2\right) d_{G}(u)=M_{1}(G)-4 m
$$

which is the desired result. In the above inequality, equality holds if and only if all the vertices of $G$ are of degree more than one.

Next, we shall determine the irregularity of the graph $R(G)$ in terms of the number of edges, the first Zagreb index and the irregularity of the original graph $G$.

Theorem 2 The irregularity of the triangle parallel graph $R(G)$ of $G$ is given by

$$
\operatorname{irr}(R(G))=2 \operatorname{irr}(G)+2 M_{1}(G)-4 m .
$$

Proof From the definition of triangle parallel graph $R(G)$ of $G$, it is clear that, $d_{R(G)}(v)=$ $2 d_{G}(v)$, if $v \in V(G)$, and $d_{R(G)}(v)=2$, if $v \in V(R(G)) \backslash V(G)$. So, $|V(R(G))|=n+m$ and $|E(R(G))|=3 m$. Hence, from (1) the irregularity of $R(G)$ is given by

$$
\begin{aligned}
\operatorname{irr}(R(G))= & \sum_{u v \in E(R(G))}\left|d_{R(G)}(u)-d_{R(G)}(v)\right| \\
= & \sum_{\substack{u, v \in V(G) \\
u v \in E(R(G))}}\left|d_{R(G)}(u)-d_{R(G)}(v)\right| \\
& +\sum_{\substack{p \in V(G) \\
q \in V(R(G)) \backslash V(G) \\
p q \in E(R(G))}}\left|d_{R(G)}(p)-d_{R(G)}(q)\right| \\
= & \sum_{u v \in E(G)}\left|2 d_{G}(u)-2 d_{G}(v)\right|+\sum_{p \in V(G)}\left|2 d_{G}(p)-2\right| d_{G}(p) \\
= & 2 \sum_{u v \in E(G)}\left|d_{G}(u)-d_{G}(v)\right|+2 \sum_{p \in V(G)}\left(d_{G}(p)-1\right) d_{G}(p)
\end{aligned}
$$

from where the desired result follows. 
Now, we shall determine the irregularity of $Q(G)$ in terms of the first Zagreb index of the graph $G$ and the irregularity of the line graph of $G$.

Theorem 3 The irregularity of the line superposition graph $Q(G)$ of $G$ is given by

$$
\operatorname{irr}(Q(G))=\operatorname{irr}(L(G))+M_{1}(G)
$$

where $L(G)$ is the line graph of $G$.

Proof From the construction of $Q(G)$ it is clear that, $|V(Q(G))|=n+m$ and $|E(Q(G))|=$ $2 m+|E(L(G))|$. Also, $d_{Q(G)}(v)=d_{G}(v)$, if $v \in V(G)$, and $d_{Q(G)}(v)=d_{L(G)}(v)+2$, if $v \in V(R(G)) \backslash V(G)$. Hence, from (1), the irregularity of $Q(G)$ is given by

$$
\begin{aligned}
& \operatorname{irr}(Q(G))=\sum_{u v \in E(Q(G))}\left|d_{Q(G)}(u)-d_{Q(G)}(v)\right| \\
& =\sum_{\substack{u \in V(G) \\
v \in V(Q(G)) \backslash V(G) \\
u v \in E(Q(G))}}\left|d_{Q(G)}(u)-d_{Q(G)}(v)\right|+\sum_{\substack{p \in V(Q(G)) \backslash V(G) \\
q \in V(Q(G)) \backslash V(G) \\
p q \in E(Q(G))}}\left|d_{Q(G)}(p)-d_{Q(G)}(q)\right| \\
& =\sum_{\substack{u \in V(G) \\
u v \in E(G)}}\left|d_{G}(u)-\left\{d_{G}(u)+d_{G}(v)\right\}\right| \\
& +\sum_{\substack{p \in V(Q(G)) \backslash V(G) \\
q \in V(Q(G)) \backslash V(G) \\
p q \in E(Q(G))}}\left|d_{L(G)}(p)+2-d_{L(G)}(q)-2\right| \\
& =\sum_{u \in V(G)} d_{G}(u)^{2}+\sum_{p q \in E(L(G))}\left|d_{L(G)}(p)-d_{L(G)}(q)\right| \\
& =\operatorname{irr}(L(G))+M_{1}(G),
\end{aligned}
$$

which is the desired result.

Finally, we express the irregularity of the total graph $T(G)$ of $G$ in terms of the irregularity of the original graph $G$ and that of the line graph of $G$.

Theorem 4 The irregularity of the total graph $T(G)$ of $G$ is given by

$$
\operatorname{irr}(T(G))=\operatorname{irr}(L(G))+4 \operatorname{irr}(G)
$$

where $L(G)$ is the line graph of $G$.

Proof For the total graph $T(G), V(T(G))=V(G) \cup E(G)$ and any two vertices of $T(G)$ are adjacent if and only if, the corresponding elements of $G$ are either adjacent or incident. So, $|V(T(G))|=n+m$ and $|E(T(G))|=2 m+|E(L(G))|$. For the total graph, it is clear that $d_{T(G)}(v)=2 d_{G}(v)$, if $v \in V(G)$ and $d_{T(G)}(v)=d_{L(G)}(v)+2$, if $v \in V(T(G)) \backslash V(G)$. Therefore, from (1), the irregularity of the total graph $T(G)$ is given by

$$
\begin{aligned}
\operatorname{irr}(T(G)) & =\sum_{u v \in E(T(G))}\left|d_{T(G)}(u)-d_{T(G)}(v)\right| \\
& =\sum_{\substack{u, v \in V(G) \\
u v \in E(T(G))}}\left|d_{T(G)}(u)-d_{T(G)}(v)\right|+\sum_{\substack{u \in V(G) \\
v \in V(T(G)) \backslash V(G) \\
u v \in E(T(G))}}\left|d_{T(G)}(u)-d_{T(G)}(v)\right|
\end{aligned}
$$




$$
\begin{aligned}
& +\sum_{\substack{u \in V(T(G)) \backslash V(G) \\
v \in V(T(G))(V(G) \\
u v \in E(T(G))}}\left|d_{T(G)}(u)-d_{T(G)}(v)\right| \\
= & \sum_{\substack{u, v \in V(G) \\
u v \in E(G)}}\left|2 d_{G}(u)-2 d_{G}(v)\right|+\sum_{\substack{u, v \in V(G) \\
u v \in E(G)}}\left|2 d_{G}(u)-\left\{d_{G}(v)+d_{G}(v)\right\}\right| \\
& +\sum_{\substack{u, v \in V(L(G)) \\
u v \in E(L(G))}}\left|d_{L(G)}(u)-d_{L(G)}(v)\right| \\
= & 2 \sum_{\substack{u v \in E(G) \\
u v\left(d_{G}(u)-d_{G}(v) \mid+2\right.}}\left|\sum_{u v \in E(G)}\right| d_{G}(u)-d_{G}(v) \mid+\operatorname{irr}(L(G)) \\
= & \operatorname{irr}(G)+\operatorname{irr}(L(G)),
\end{aligned}
$$

which is the desired result.

Example 1 Let $K_{m, n}$ be the complete bipartite graph where $m, n \geq 2$ and $P_{n}$ be a path on $n$ vertices $(n \geq 2)$. The following results are obtained as direct consequences of Theorems 1 , 2,3 and 4 .

(i) $\operatorname{irr}\left(S\left(K_{m, n}\right)\right)=m n(m+n-4)$;

(ii) $\operatorname{irr}\left(R\left(P_{n}\right)\right)=4(n-1)=\operatorname{irr}\left(Q\left(P_{n}\right)\right)$; and

(iii) $\operatorname{irr}\left(T\left(P_{n}\right)\right)=10$.

\section{Irregularity of Double Graph and Extended Double cover}

In this section, we give exact expressions of irregularity of double graph and extended double cover in terms of that of the original graph.

Let us denote the double graph of a graph $G$ by $G^{*}$, which is constructed from two copies of $G$ in the following manner $[3,10,17]$. Let the vertex set of $G$ be $V(G)=\left\{v_{1}, v_{2}, \ldots, v_{n}\right\}$, and the vertices of $G^{*}$ are given by the two sets $X=\left\{x_{1}, x_{2}, \ldots, x_{n}\right\}$ and $Y=\left\{y_{1}, y_{2}, \ldots, y_{n}\right\}$. Thus for each vertex $v_{i} \in V(G)$, there are two vertices $x_{i}$ and $y_{i}$ in $V\left(G^{*}\right)$. The double graph $G^{*}$ includes the initial edge set of each copies of $G$, and for any edge $v_{i} v_{j} \in E(G)$, two more edges $x_{i} y_{j}$ and $x_{j} y_{i}$ are added. The double graph of the path $P_{3}$ is illustrated in Fig. 1.

Theorem 5 The irregularity of the double graph $G^{*}$ of a graph $G$ is given by

$$
\operatorname{irr}\left(G^{*}\right)=\operatorname{sirr}(G) .
$$

Proof From the definition of double graph it is clear that $d_{G^{*}}\left(x_{i}\right)=d_{G^{*}}\left(y_{i}\right)=2 d_{G}\left(v_{i}\right)$, where $v_{i} \in V(G)$ and $x_{i}, y_{i} \in V\left(G^{*}\right)$ are corresponding clone vertices of $v_{i}$. Thus the irregularity of double graph $G^{*}$ is
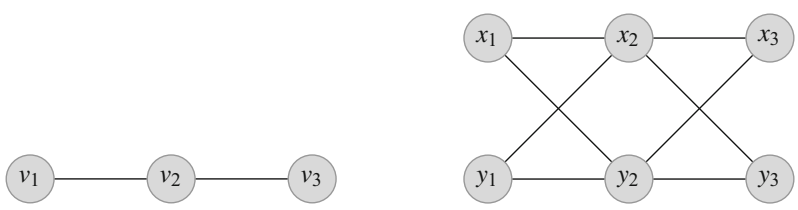

Fig. 1 The graph $P_{3}$ and its double graph $P_{3}^{*}$ 


$$
\begin{aligned}
\operatorname{irr}\left(G^{*}\right)= & \sum_{u v \in E\left(G^{*}\right)}\left|d_{G^{*}}(u)-d_{G^{*}}(v)\right| \\
= & \sum_{x_{i} x_{j} \in E\left(G^{*}\right)}\left|d_{G^{*}}\left(x_{i}\right)-d_{G^{*}}\left(x_{j}\right)\right|+\sum_{y_{i} y_{j} \in E\left(G^{*}\right)}\left|d_{G^{*}}\left(y_{i}\right)-d_{G^{*}}\left(y_{j}\right)\right| \\
& +\sum_{x_{i} y_{j} \in E\left(G^{*}\right)}\left|d_{G^{*}}\left(x_{i}\right)-d_{G^{*}}\left(y_{j}\right)\right|+\sum_{x_{j} y_{i} \in E\left(G^{*}\right)}\left|d_{G^{*}}\left(x_{j}\right)-d_{G^{*}}\left(y_{i}\right)\right| \\
= & 4 \sum_{v_{i} v_{j} \in E(G)}\left|2 d_{G}\left(v_{i}\right)-2 d_{G}\left(v_{j}\right)\right|,
\end{aligned}
$$

from where the desired result follows.

Let $G=(V, E)$ be a simple connected graph with $V=\left\{v_{1}, v_{2}, \ldots, v_{n}\right\}$. The extended double cover of $G$, denoted by $G^{* *}$ is the bipartite graph with bipartition $(X, Y)$ where $X=\left\{x_{1}, x_{2}, \ldots, x_{n}\right\}$ and $Y=\left\{y_{1}, y_{2}, \ldots, y_{n}\right\}$ in which $x_{i}$ and $y_{j}$ are adjacent if and only if either $v_{i}$ and $v_{j}$ are adjacent in $G$ or $i=j$. For example, the extended double cover of the complete graph is the complete bipartite graph. This construction of the extended double cover was introduced by Alon [3] in 1986. In Fig. 2, we illustrate the extended double cover of $P_{3}$.

Theorem 6 The irregularity of the extended double cover $G^{* *}$ of the graph $G$ is given by

$$
\operatorname{irr}\left(G^{* *}\right)=2 \operatorname{irr}(G) .
$$

Proof If $G$ is a graph with $n$ vertices and $m$ edges, then from definition of extended double cover graph $G^{* *}$ consists of $2 n$ vertices and $(n+2 m)$ edges and $d_{G^{* *}}\left(x_{i}\right)=d_{G^{* *}}\left(y_{i}\right)=$ $d_{G}\left(v_{i}\right)+1$, for $i=1,2, \ldots, n$. Here, $v_{i} \in V(G)$ and $x_{i}, y_{i} \in V\left(G^{* *}\right)$ are corresponding clone vertices of $v_{i}$. Thus the irregularity of extended double cover graph $G^{* *}$ of $G$ is given by

$$
\begin{aligned}
\operatorname{irr}\left(G^{* *}\right)= & \sum_{u v \in E\left(G^{* *}\right)}\left|d_{G^{* *}}(u)-d_{G^{* *}}(v)\right| \\
= & \sum_{x_{i} y_{j} \in E\left(G^{* *}\right)}\left|d_{G^{* *}}\left(x_{i}\right)-d_{G^{* *}}\left(y_{j}\right)\right| \\
& +\sum_{x_{j} y_{i} \in E\left(G^{* *}\right)}\left|d_{G^{* *}}\left(x_{j}\right)-d_{G^{* *}}\left(y_{i}\right)\right|+\sum_{i=1}^{n}\left|d_{G^{* *}}\left(x_{i}\right)-d_{G^{* *}}\left(y_{i}\right)\right| \\
= & 2 \sum_{v_{i} v_{j} \in E(G)}\left|d_{G}\left(v_{i}\right)+1-d_{G}\left(v_{j}\right)-1\right|,
\end{aligned}
$$

from where the desired result follows.
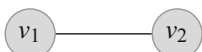

$v_{3}$

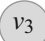

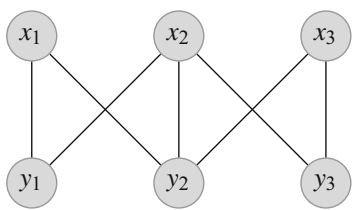

Fig. 2 The graph $P_{3}$ and its extended double cover $P_{3}^{* *}$ 


\section{Irregularity of Thorn Graph}

An edge $e=u v$ of a graph $G$ is called a thorn if either $d(u)=1$ or $d(v)=1$. The concept of thorn graph was first introduced by Gutman [14] by joining a number of thorn to each vertex of any given graph $G$. A variety of topological indices of thorn graphs have been already studied by the researchers, in the recent past [5-8, 16, 18, 23, 25,26]. In this section, our aim is to study irregularity of graphs in terms of some auxiliary invariants.

Let $V(G)$ and $V\left(G^{T}\right)$ be the vertex sets of $G$ and its thorn graph $G^{T}$ respectively. Let $V(G)=\left\{v_{1}, v_{2}, \ldots, v_{n}\right\}$ and $V^{T}(G)=V(G) \cup V_{1} \cup V_{2} \cup \ldots \cup V_{n}$, where $V_{i}$ are the set of degree one vertices attached to the vertices $v_{i}$ in $G^{T}$ and $V_{i} \cap V_{j}=\varphi, i \neq j$. Let the vertices of the set $V_{i}$ are denoted by $v_{i j}$ for $j=1,2, \ldots, p_{i}$ and $i=1,2, \ldots, n$. Thus $\left|V\left(G^{T}\right)\right|=n+z$ where, $z=\sum_{i=1}^{n} p_{i}$. Then the degree of the vertices $v_{i}$ in $G^{T}$ are given by $d_{G^{T}}\left(v_{i}\right)=d_{G}\left(v_{i}\right)+p_{i}$, for $i=1,2, \ldots, n$. In the following, we first find the irregularity of the general thorn graph $G^{T}$ and then consider some particular cases.

Theorem 7 The irregularity of a thorn graph $G^{T}$ is given by

$$
\operatorname{irr}\left(G^{T}\right) \leq \operatorname{irr}(G)+\sum_{i=1}^{n} p_{i} d_{G}\left(v_{i}\right)+\sum_{i=1}^{n} p_{i}{ }^{2}+\sum_{v_{i} v_{j} \in E(G)}\left|p_{i}-p_{j}\right|-z .
$$

Proof From (1), the irregularity of the thorn graph $G^{T}$ is given by

$$
\begin{aligned}
& \sum_{v_{i} v_{j} \in E\left(G^{T}\right)}\left|d_{G^{T}}\left(v_{i}\right)-d_{G^{T}}\left(v_{j}\right)\right| \\
= & \sum_{v_{i} v_{j} \in E(G)}\left|d_{G^{T}}\left(v_{i}\right)-d_{G^{T}}\left(v_{j}\right)\right|+\sum_{i=1}^{n} \sum_{j=1}^{p_{i}}\left|d_{G^{T}}\left(v_{i}\right)-d_{G^{T}}\left(v_{i j}\right)\right| \\
= & \sum_{v_{i} v_{j} \in E(G)}\left|d_{G}\left(v_{i}\right)+p_{i}-d_{G^{T}}\left(v_{j}\right)-p_{j}\right|+\sum_{i=1}^{n} \sum_{j=1}^{p_{i}}\left|d_{G}\left(v_{i}\right)+p_{i}-1\right| \\
\leq & \sum_{v_{i} v_{j} \in E(G)}\left|d_{G}\left(v_{i}\right)-d_{G}\left(v_{j}\right)\right|+\sum_{v_{i} v_{j} \in E(G)}^{n}\left|p_{i}-p_{j}\right|+\sum_{i=1}^{n} p_{i}\left\{d_{G}\left(v_{i}\right)+p_{i}-1\right\} \\
= & \operatorname{irr}(G)+\sum_{v_{i} v_{j} \in E(G)}\left|p_{i}-p_{j}\right|+\sum_{i=1}^{n} p_{i} d_{G}\left(v_{i}\right)+\sum_{i=1}^{n} p_{i}{ }^{2}-\sum_{i=1}^{n} p_{i} .
\end{aligned}
$$

from where the desired result follows.

The following corollaries are direct consequences of the previous theorem.

Corollary 1 Let $G^{T}$ be a thorn graph with parameters $p_{i}=t$ for all $i$, then

$$
\operatorname{irr}\left(G^{T}\right)=\operatorname{irr}(G)+t(2|E(G)|+n(t-1)) .
$$

Corollary 2 If the parameters $p_{i}(\geq 1)$ is equal to the degree of the corresponding vertex $v_{i}$, then

$$
\operatorname{irr}\left(G^{T}\right)=2\left[\operatorname{irr}(G)+M_{1}(G)-|E(G)|\right] .
$$


Corollary 3 If $\lambda$ be an integer so that $\lambda>d_{G}\left(v_{i}\right), i=1,2, \ldots, n$ and if $G^{T}$ be the thorn graph with parameters $p_{i}=\lambda-d_{G}\left(v_{i}\right)$, then

$$
\operatorname{irr}\left(G^{T}\right)=(\lambda-1)(n \lambda-2|E(G)|) .
$$

Corollary 4 If the number of thorns, i.e., pendant edges attached to any vertex of the parent graph is a linear function of the degree of the corresponding vertex $v_{i}$, i.e., $p_{i}=a d_{G}\left(v_{i}\right)+b$, where $a$ and $b$ are any constants, the irregularity of the thorn graph is given by

$$
\operatorname{irr}\left(G^{T}\right)=(a+1) \operatorname{irr}(G)+a(a+1) M_{1}(G)+2(b-a+2 a b)|E(G)|+b n(b-1) .
$$

Corollary 5 Let $C_{n}^{T}$ be the thorny ring (having $n$ ring as parent and $(z-2)$ thorns at each vertex) then $\operatorname{irr}\left(C_{n}^{T}\right)=n(z-1)(z-2)$.

Corollary 6 Let $P_{n}^{T}$ be the thorn path (caterpillar) obtained from $P_{n}$ by attaching z pendent vertices at each vertex of $P_{n}$, then we have $\operatorname{irr}\left(P_{n}^{T}\right)=n z^{2}+(n-2) z$.

Corollary 7 Let $S_{n}^{T}$ be the thorn star obtained from $S_{n}\left(\cong K_{1, n}\right)$ by joining $z$ thorns at each vertex of the parent graph $S_{n}$. Then, $\operatorname{irr}\left(S_{n}^{T}\right)=(n+1) z^{2}+(n-1)(z+n)$.

\section{Irregularity of Subdivision Vertex Corona of Graphs}

In this section,we give exact expression for irregularity of subdivision vertex corona of two graphs and then consider some particular cases.

Let $G_{1}$ and $G_{2}$ be any two simple connected graph with $n_{1}$ and $n_{2}$ number of vertices and $m_{1}$ and $m_{2}$ number of edges respectively.The subdivision vertex corona of $G_{1}$ and $G_{2}$ is denoted by $G_{1} \odot G_{2}$ and was introduced by Lu and Miao [19]. The graph $G_{1} \odot G_{2}$ is obtained from $S\left(G_{1}\right)$ and $n_{1}$ copies of $G_{2}$, by joining the $i$-th vertex of $V\left(G_{1}\right)$ to every vertex in the $i$-th copy of $G_{2}$. Let $V\left(G_{1}\right)=\left\{v_{1}, v_{2}, \ldots, v_{n_{1}}\right\}, I\left(G_{1}\right)=\left\{v_{1}^{e}, v_{2}^{e}, \ldots, v_{m_{1}}^{e}\right\}$ and $V\left(G_{2}\right)=\left\{u_{1}, u_{2}, \ldots, u_{n_{2}}\right\}$, so that $V(S(G))=V(G) \cup I(G)$. Let $u_{1}^{i}, u_{2}^{i}, \ldots, u_{n_{2}}^{i}$ denote the vertices of the $i$-th copy of $G_{2, i}, i=1,2, \ldots, n_{1}$, so that

$$
V\left(G_{1} \odot G_{2}\right)=V\left(G_{1}\right) \cup I\left(G_{1}\right) \cup\left[V\left(G_{2,1}\right) \cup V\left(G_{2,2}\right) \cup \ldots \cup V\left(G_{2, n_{1}}\right)\right] .
$$

Theorem 8 The irregularity of $G_{1} \odot G_{2}$ is given by

$$
\operatorname{irr}\left(G_{1} \odot G_{2}\right)=n_{1} \operatorname{irr}\left(G_{2}\right)+M_{1}\left(G_{1}\right)+4 m_{1} n_{2}-2 n_{1} m_{2}+n_{1} n_{2}\left(n_{2}-1\right)-4 m_{1} .
$$

Proof The degree of the vertices of $G_{1} \odot G_{2}$ is given by $d_{G_{1} \odot G_{2}}\left(v_{i}\right)=d_{G_{1}}\left(v_{i}\right)+n_{2}$ for $i=1,2, \ldots, n_{1}, d_{G_{1} \odot G_{2}}\left(e_{i}\right)=2$ for $i=1,2, \ldots, m_{1}, d_{G_{1} \odot G_{2}}\left(u_{j}^{i}\right)=d_{G_{2}}\left(u_{j}\right)+1$ for $i=1,2, \ldots, n_{1}$ and $j=1,2, \ldots, n_{2}$. Let the vertex set of $G_{1} \odot G_{2}$ can be partitioned into three subsets

$$
\begin{aligned}
& E_{1}=\left\{x y \in E\left(G_{1} \odot G_{2}\right): x, y \in V\left(G_{2, i}\right), i=1,2, \ldots, n_{1}\right\}, \\
& E_{2}=\left\{x y \in E\left(G_{1} \odot G_{2}\right): x \in V\left(G_{1}\right), y \in I\left(G_{1}\right)\right\}, \text { and } \\
& E_{3}=\left\{x y \in E\left(G_{1} \odot G_{2}\right): x \in V\left(G_{1}\right), y \in V\left(G_{2, i}\right), i=1,2, \ldots, n_{1}\right\} .
\end{aligned}
$$

The contribution of the edges in $E_{1}$ to the irregularity of $G_{1} \odot G_{2}$ is given by

$$
\begin{aligned}
\operatorname{irr}_{1}\left(G_{1} \odot G_{2}\right) & =\sum_{x y \in E_{1}}\left|d_{G_{1} \odot G_{2}}(x)-d_{G_{1} \odot G_{2}}(y)\right| \\
& =\sum_{i=1}^{n_{1}} \sum_{u_{i} u_{j} \in E\left(G_{2}\right)}\left|d_{G_{2}}\left(u_{i}\right)+1-d_{G_{2}}\left(u_{j}\right)-1\right|=n_{1} \operatorname{irr}\left(G_{2}\right) .
\end{aligned}
$$


Similarly, the contribution of the edges in $E_{2}$ to the irregularity of $G_{1} \odot G_{2}$ is given by

$$
\begin{aligned}
\operatorname{irr}_{2}\left(G_{1} \odot G_{2}\right) & =\sum_{x y \in E_{1}}\left|d_{G_{1} \odot G_{2}}(x)-d_{G_{1} \odot G_{2}}(y)\right| \\
& =\sum_{i=1}^{n_{1}}\left|d_{G_{1}}\left(v_{i}\right)+n_{2}-2\right| d_{G_{1}}\left(v_{i}\right) \\
& =\sum_{i=1}^{n_{1}} d_{G_{1}}\left(v_{i}\right)^{2}+\left(n_{2}-2\right) \sum_{i=1}^{n_{1}} d_{G_{1}}\left(v_{i}\right) \\
& =M_{1}\left(G_{1}\right)+2\left(n_{2}-2\right) m_{1} .
\end{aligned}
$$

Also, for the edges in $E_{3}$, contribution to the irregularity of $G_{1} \odot G_{2}$ is given by

$$
\begin{aligned}
\operatorname{irr}_{3}\left(G_{1} \odot G_{2}\right) & =\sum_{x y \in E_{3}}\left|d_{G_{1} \odot G_{2}}(x)-d_{G_{1} \odot G_{2}}(y)\right| \\
& =\sum_{i=1}^{n_{1}} \sum_{j=1}^{n_{2}}\left|d_{G_{1}}\left(v_{i}\right)+n_{2}-d_{G_{2}}\left(u_{j}\right)-1\right| \\
& =n_{1} n_{2}\left(n_{2}-1\right)+2 n_{2} m_{1}-2 n_{1} m_{2} .
\end{aligned}
$$

The desired expression for the irregularity of $G_{1} \odot G_{2}$ is obtained by summing the above three expressions.

Let $|V(G)|=p$ and $|E(G)|=q$, then the following corollaries are direct consequence of Theorem 8.

Corollary 8 Let $C_{n}$ be cycle on $n$ vertices. Then for any simple graph $G$,

(i) $\operatorname{irr}\left(C_{n} \odot G\right)=n \operatorname{irr}(G)+n p^{2}+3 n p-2 n q$,

(ii) $\operatorname{irr}\left(G \odot C_{n}\right)=M_{1}(G)+n p^{2}-3 n p+4 n q-4 q$.

Corollary 9 Let $P_{n}$ be path on $n$ vertices. Then for any simple graph $G$,

(i) $\operatorname{irr}\left(P_{n} \odot G\right)=n \operatorname{irr}(G)+n p^{2}+3 n p-2 n q-4 p-2$,

(ii) $\operatorname{irr}\left(G \odot P_{n}\right)=M_{1}(G)+n p^{2}-3 n p+4 n q+4 p-4 q$.

Using Corollary 8 and 9 the following results are obtained by straight forward calculations.

Example 2 (i) $\operatorname{irr}\left(C_{n} \odot C_{m}\right)=n m^{2}+n m$;

(ii) $\operatorname{irr}\left(P_{n} \odot P_{m}\right)=n m^{2}+n m+4 n-4 m-2$;

(iii) $\operatorname{irr}\left(C_{n} \odot P_{m}\right)=n m^{2}+n m+4 n$; and

(iv) $\operatorname{irr}\left(P_{n} \odot C_{m}\right)=n m^{2}+n m-4 m-2$.

Acknowledgments The authors thanks the anonymous reviewers whose comments helped improve and clarify this manuscript. 


\section{References}

1. Abdo, H., Dimitrov, D.: The irregularity of graphs under graph operations. Discuss. Math. Graph Theo. 34(2), 263-278 (2014)

2. Albertson, M.O.: The Irregularity of a graph. Ars Comb. 46, 219-225 (1997)

3. Alon, N.: Eigenvalues and expanders. Combinatorica 6, 83-96 (1986)

4. Bell, F.K.: A note on the irregularity of graphs. Linear Algebra Appl. 161, 45-54 (1992)

5. Bonchev, D., Klein, D.J.: On the Wiener number of thorn trees, stars, rings, and rods. Croat. Chem. Acta 75, 613-620 (2002)

6. De, N.: On eccentric connectivity index and polynomial of thorn graph. Appl. Math. 3, 931-934 (2012)

7. De, N.: Augmented eccentric connectivity index of some thorn graphs. Intern. J. Appl. Math. Res. 1(4), 671-680 (2012)

8. De, N., Pal, A., Nayeem, S.M.A.: Modified eccentric connectivity of generalized thorn graphs. Intern. J. Comput. Math. doi:10.1155/2014/436140

9. De, N., Nayeem, S.M.A., Pal, A.: Total eccentricity index of the generalized hierarchical product of graphs. Intern. J. Appl. Comput. Math. doi:10.1007/s40819-014-0016-4

10. Dehghan-Zadeh, T., Hua, H., Ashrafi, A.R., Habibi, N.: Remarks on a conjecture about Randić index and graph radius. Miskolc Math. Notes 14(3), 845-850 (2013)

11. Eliasi, M., Taeri, B.: Four new sums of graphs and their Wiener indices. Discret. Appl. Math. 157, 794-803 (2009)

12. Fath-Tabar, G.H.: Old and new Zagreb indices of graphs. MATCH Commun. Math. Comput. Chem. 65, 79-84 (2011)

13. Gutman, I., Trinajstić, N.: Graph theory and molecular orbitals. Total $\pi$-electron energy of alternant hydrocarbons. Chem. Phys. Lett. 17, 535-538 (1972)

14. Gutman, I.: Distance in thorny graph. Publ. Inst. Math. 63, 31-36 (1998)

15. Hansen, P., Mélot, H.: Variable neighborhood search for extremal graphs 9 . Bounding the irregularity of a graph. DIMACS Ser. Discret. Math. Theo. Comput. Sci. 69, 253-264 (1962)

16. Heydari, A., Gutman, I.: On the terminal Wiener index of thorn graphs. Kragujevac J. Sci. 32, 57-64 (2010)

17. Hua, H., Zhang, S., Xu, K.: Further results on the eccentric distance sum. Discret. Appl. Math. 160, 170-180 (2012)

18. Kathiresan, K.M., Parameswaran, C.: Certain generalized thorn graphs and their Wiener indices. J. Appl. Math. Inform. 30(5-6), 793-807 (2012)

19. Lu, P., Miao, Y.: Spectra of the subdivision-vertex and subdivision-edge coronae, arXiv:1302.0457v2 (2013)

20. Nasiri, R., Fath-Tabar, G.H.: The second minimum of the irregularity of graphs. Electron. Notes Discret. Math. 45, 133-140 (2014)

21. Réti, T., Dimitrov, D.: On irregularities of bidegreed graphs. Acta Polytech. Hung. 10, 117-134 (2013)

22. Tavakoli, M., Rahbarnia, F., Ashrafi, A.R.: Some new results on irregularity of graphs. J. Appl. Math. Inform. 32, 675-685 (2014)

23. Walikar, H.B., Ramane, H.S., Sindagi, L., Shirakol, S.S., Gutman, I.: Hosoya polynomial of thorn trees, rods, rings, and stars. Kragujevac J. Sci. 28, 47-56 (2006)

24. Yan, W., Yang, B.Y., Yeh, Y.N.: The behavior of Wiener indices and polynomials of graphs under five graph decorations. Appl. Math. Lett. 20, 290-295 (2007)

25. Zhou, B.: On modified Wiener indices of thorn trees. Kragujevac J. Math. 27, 5-9 (2005)

26. Zhou, B., Vukičevič, D.: On Wiener-type polynomials of thorn graphs. J. Chemom. 23, 600-604 (2009) 